\title{
Role of Corticosteroids in Influenza Pneumonia (H1N1) Patients
}

\author{
Krupanidhi Karunanithi ${ }^{1, *}$, Ajith $\mathbf{V}^{1}$, Ajith Kumar.J ${ }^{1}$, Naveen Mohan ${ }^{1}$, Arun Kumar ${ }^{1}$, Elson Kuriakose ${ }^{1}$, \\ Deepan Kumar ${ }^{2}$, S.Kavitha ${ }^{3}$, Sreekrishnan T.P ${ }^{1}$, Gireesh Kumar ${ }^{1}$ \\ ${ }^{1}$ Department of Emergency Medicine, Amrita Institute of Medical Science and Research, India \\ ${ }^{2}$ Department of Community Dentistry, Amrita Institute of Dental Science and Research, India \\ ${ }^{3}$ Vivekananda Institute of Information and Management Studies, India
}

Copyright $(2015$ by authors, all rights reserved. Authors agree that this article remains permanently open access under the terms of the Creative Commons Attribution License 4.0 International License

\begin{abstract}
Background and objective: Although the benefits of systemic corticosteroids in pneumonia are not clear, their use is frequent in clinical practice. We described the patients' characteristics, demographics and the effect of steroids in Influenza pneumonia Hemagglutinin Type 1 and Neuraminidase Type 1 (H1N1) patients in terms of length of hospital stay. METHODS: The study design was a retrospective observational study, which consisted of patients presenting with $\mathrm{H} 1 \mathrm{~N} 1$ infection to the department of Emergency medicine at tertiary referral centre. We investigated all cases admitted with diagnosis of H1N1 pneumonia, confirmed by collecting throat swabs and doing Reverse Transcriptase Polymerase Chain Reaction (RT-PCR) for H1N1 infection during July 2009 to February 2011. RESULTS: Of the 115 patients, 30 patients have been administered steroids. Within those 30 patients 20 have stayed in ICU with mean $7.45 \pm 5.7$ days. In that group of 20 patients, 10 patients have stayed in ICU alone, 10 patients have stayed in ICU and ward with mean $10.3 \pm 6.5$ and $13.6 \pm 7.4$ days respectively. 10 patients have stayed only in ward with mean days of $4.8 \pm 1.9$. The overall mean hospital stay is $9.8 \pm 6.6$ days. It is found that there is a significant statistical relationship between corticosteroid administration and reduced hospital stay $(\mathrm{P}=0.000)$.
\end{abstract}

Keywords H1N1, Pneumonia, Corticosteroids, Swine Flu

\section{Introduction}

Community-acquired pneumonia (CAP) is the leading cause of morbidity and mortality from infectious diseases in developed countries. It affects more than 5 million adults and accounts for more than 1 million admissions each year in the United States. Pneumonia is the sixth leading cause of death worldwide, and age-adjusted mortality is increasing [1].
H1N1 Influenza is the subtype of influenza A virus that was responsible for the pandemic which occurred during 2009. Influenza $\mathrm{A}$ is a re-assorted influenza virus reported from Mexico and rapidly spread to neighbouring countries United States and Canada. Subsequently the disease spread to all the continents. The current virus represents a quadruple re-assortment of two swine strains, one human strain and one avian strain of influenza; the largest proportion of genes coming from swine influenza virus[2]. Swine influenza (swine flu or pig flu) is a respiratory disease that occurs in pigs, that is caused by influenza A virus and spreads to humans. A confirmed case of pandemic H1N1 influenza is defined as an individual with influenza like illness with laboratory-confirmed H1N1 influenza a virus detected by Reverse Transcriptase Polymerase Chain Reaction (RT-PCR). Influenza-like illness is defined as fever (temperature of $100 \mathrm{~F}$ [37.8] or greater) with cough or sore throat in the absence of a known cause other than influenza.

In India, the first case of influenza A H1N1 was reported on May 16, 2009 and the first case confirmed on 16 May 2009, when a man travelling from New York via Dubai and Delhi tested positive for the H1N1 Influenza virus in Hyderabad. The Novel H1N1 caused serious fatal infection in normal individuals but pregnant women, elderly, younger children and people of any age with certain chronic lung or other medical conditions appear to be at higher risk of more complicated infection or fatal outcome [24]. During the 2009-2010 wave of the pandemic, Kerala was affected as much as the other states of India, according to the data provided by the H1N1 Cell of the Health Services Department of Kerala. Kerala was one of the worst affected states in India, most probably due to the population density and the overcrowding in the cities. The first case in Kerala was reported on June $24^{\text {th }}$, 2009, from Ernakulum District, imported from the United Kingdom. During the $2^{\text {nd }}$ wave in 2010, Kerala and Maharashtra were the 2 states predominantly hit by the pandemic resurgence. The disease was deemed 'notifiable' on August 26th, 2009, by the 
Government of Kerala. Since Kerala had faced major threats during both the waves of infection, a study on the spread of this novel virus in the state's population is imperative [22]

In early April 2009, a new influenza A (H1N1) 2009 virus emerged among humans in California and Mexico, quickly spreading worldwide through human-to-human transmission. By August 2010, when the transition from a pandemic to post-pandemic period was announced, 18,449 laboratory-confirmed deaths from pandemic influenza A (H1N1) 2009 had been recorded [28] (Figure 2). However, the actual number of influenza A (H1N1) cases worldwide remains unknown, as most cases was diagnosed clinically and were not confirmed in the laboratory. In most countries, the capacity for laboratory diagnosis was so stressed that diagnosis was limited to hospitalized patients [29]. However, the total number of cases of pandemic H1N1 influenza worldwide was probably on the order of several tens of millions [30, 31]. Modelling estimates of the global burden of pandemic influenza A (H1N1) 2009 ranged from several tens of millions to 2 billion. The official number of deaths from laboratory-confirmed pandemic influenza A (H1N1) 2009 infection worldwide reported to the WHO as of May 7, 2010 was at least 18,449 [28]. This number appears to be much lower than the estimated annual global mortality associated with seasonal influenza. Given the relatively high

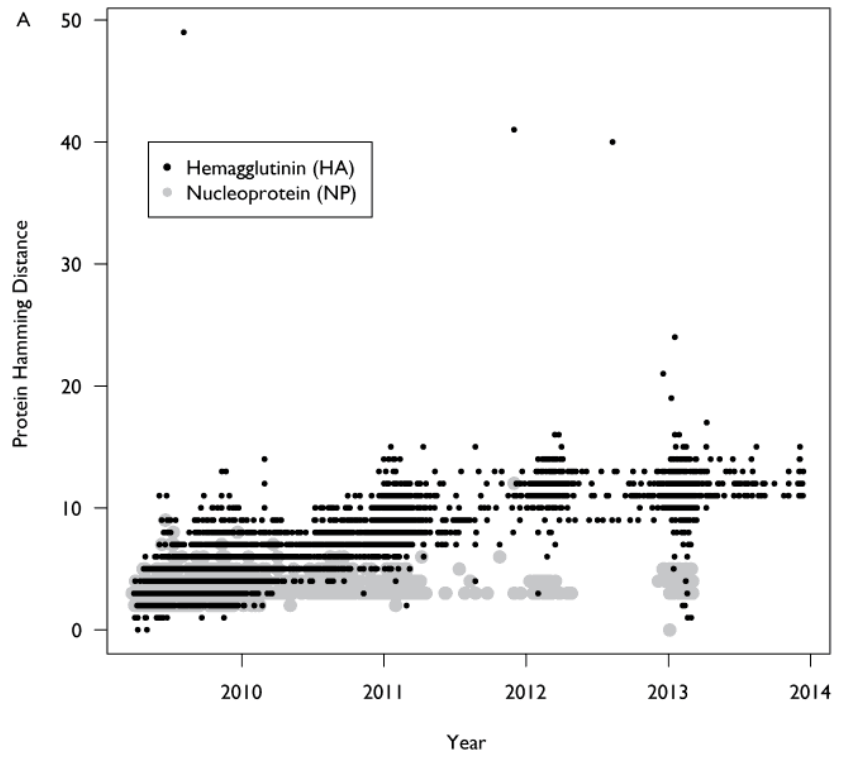

mortality rates for at-risk groups and hospitalized patients $[32,33]$, the annual mortality due to A (H1N1) 2009 is presumed to be higher.

Since April 2009, the hemagglutinin protein of the influenza H1N1 pandemic strain has been accumulating mutations at a rate of approximately 2.45 mutations per annum (A). This contrasts with the neuraminidase and nucleoprotein proteins which have been accumulating mutations at a rate of only 1.52 and 0.26 mutations per annum, respectively (B). In addition, there were more outliers present in 2013, which suggests that the population that was infected was smaller, presenting opportunities for increasing diversity (Figure 1) [34].

Corticosteroids are the most effective and widely used anti-inflammatory drugs. The role of corticosteroids as an adjunctive therapy for pneumonia is still under debate. In this course of this study we will examine their potential beneficial use of their anti-inflammatory effect and the consequent influence on pneumonia severity and outcomes. Indeed, it has been shown that the administration of systemic corticosteroids is associated with reduced pulmonary inflammation in patients with bacterial pneumonia [3]. Since there is not much study in the Indian context, this study is aimed to assess the effect of steroids in Influenza pneumonia (H1N1) patients in terms of duration of hospital stay.

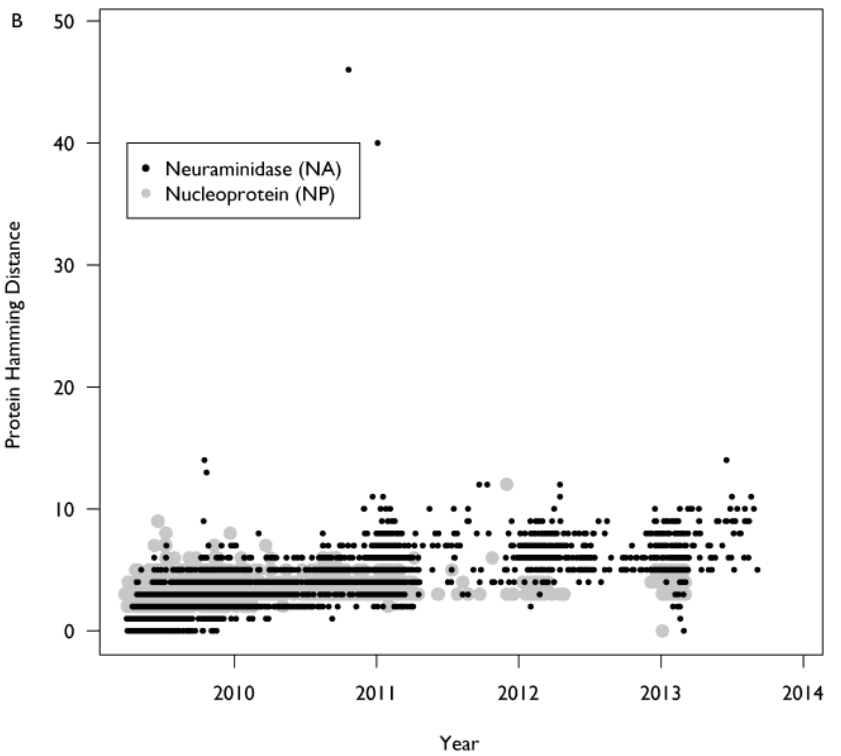

Figure 1. Protein Divergence of Influenza H1N1 Pandemic Strain, 2009-2013. 


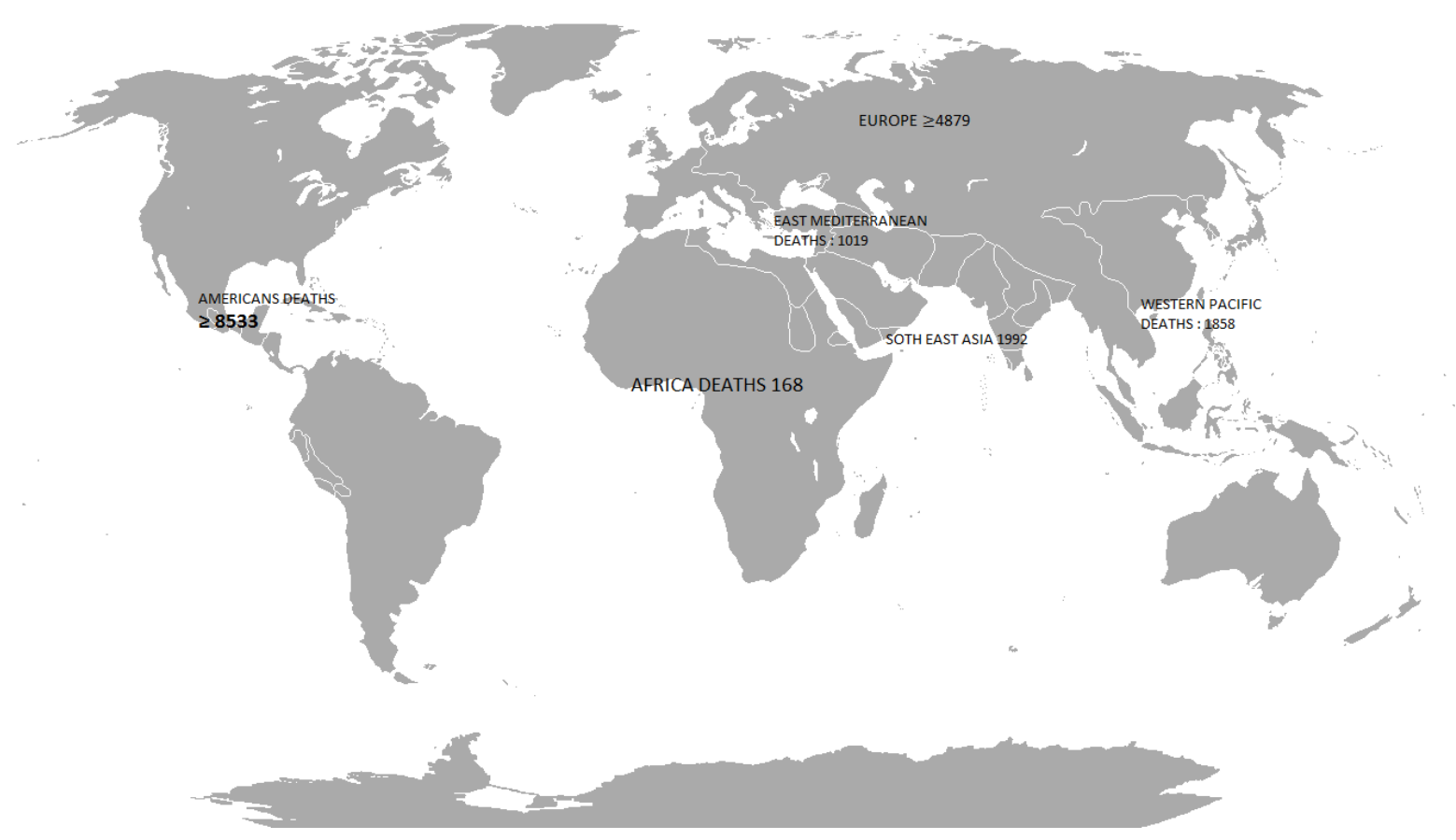

Figure 2. Areas with confirmed human deaths due to avian influenza A (H1N1) reported to the WHO, April 2009 - August 2010.

\section{Materials and Methods}

\subsection{Study Design}

The study design was a retrospective observational study, which consisted of patients presenting with H1N1 infection to the department of Emergency medicine at Amrita institute of Medical Sciences after fulfilling the inclusion and exclusion criteria.

\subsection{Sample Size}

This is a retrospective observational study on all cases admitted with diagnosis of H1N1 pneumonia, confirmed by collecting throat swabs and doing RT-PCR for H1N1 infection. Total of 115 patients were included during this study period.

\subsection{Inclusion Criteria and Exclusion Criteria}

All the cases admitted and diagnosis confirmed by throat swab RT-PCR for H1N1 infection was included. All other patients with flu like features without throat swab confirmation for H1N1 infection were excluded.

\subsection{Ethical Approval}

The proposal was reviewed and approved by the Ethical Committee, Amrita institute of Medical Sciences and Research Centre, Kochi, Kerala, India. An informed consent has been sought from the patient's bystanders.

\subsection{Methods}

A retrospective observational study was conducted in
Amrita institute of Medical Sciences and Research Centre in patients who were admitted and diagnosed to have H1N1 infection from July 2009 to February 2011. All suspected cases were confirmed by RT-PCR (by Taq Man Real Time PCR [Centre for Communicable Diseases (CDC) Protocol] for Influenza A, Swine A, Swine H1) performed at the National institute of communicable disease Delhi, Rajiv Gandhi centre for biotechnology (RGCB)Thiruvananthapuram, and KMC Manipal.

During this period the clinical profile of the H1N1 cases were analysed with reference to age distribution, sex distribution, time distribution, clinical manifestations, comorbidities, complications etc. Detailed physical examination and other investigations like complete blood count, renal function test, liver function test, and chest x-ray which were done for all patients were also analysed. Data was analysed and statistically verified by using statistical SPSS software.

Patients were identified from lists of admissions and/or laboratory results, and included if they had laboratory-confirmed influenza. Pneumonia was defined as the presence of respiratory symptoms consistent with pneumonia together with radiological evidence of consolidation. Diabetes, asthma, COPD, cystic fibrosis, chronic neurological disease and chronic liver disease, chronic renal disease was recorded as comorbidities if these diagnoses were documented in the patient notes. Immunosuppression was defined as the use of oral steroid or other immunosuppressive medication, organ transplantation, HIV infection or cancer therapy. The length of stay included the time from admission to the sentinel hospital (not including time spent at other hospitals from where they were transferred) till discharge. Duration of admission in ICU and 
ward was separately monitored. Treatment modalities like oxygen,1 Bi-level Positive Airway Pressure (BiPAP), mechanical ventilation, antibiotics, antivirals and steroids were observed.

\subsection{Laboratory Sample Collection}

Throat swabs and nasal/nasopharyngeal swabs collected by clinicians in virus transport medium (VTM) from patients exhibiting influenza like symptoms were received at the designated reference laboratory in triple packaging cold conditions. Samples were accompanied by duly filled Profromas with demographic characteristics, date of symptom onset, co-morbidities, travel/contact history, antiviral treatment, etc. Initially, all samples were collected before administration of antiviral therapy. All the respiratory specimens were processed in BSL (Bio Safety Level)-3 labs within 2-3 hours of receipt of the samples. RNA isolation was done in these samples using QIAmp ${ }^{\circledR}$ Viral RNA Mini Kit or RNeasy ${ }^{\circledR}$ Mini Kit from Qiagen, USA. Reverse transcription and amplification of the target genes was carried out by real-time RT-PCR using CDC real-time RT-PCR protocol for detection and characterization of Swine Influenza (version 2009). Primers and dual labelled probes were procured from CDC and SuperScriptTM III Platinum ${ }^{\circledR}$ one-step quantitative RT-PCR kit from Invitrogen, California, USA, was used. One-step quantitative RT-PCR probe hydrolysis (Taq Man-ABI) package kit with Agpath-IDTM and one-step RT-PCR kit supplied by Applied Biosystems-Ambion, USA, were also used later. In each sample, four target genes were amplified: Influenza A, Swine Influenza A, Swine H1 and RNaseP. A sample was declared positive when it showed amplification in all four target genes [25]-[27].

\subsection{Dosage}

Steroid therapy was initiated at a daily dose of $200 \mathrm{mg}$ hydrocortisone or equivalent doe of Methylprednisolone $125 \mathrm{mg}$, and patients were treated for a median of 1 (IQR, 0-6 days). 50 of the 83 patients received within the first 3 days and the remaining within 7 days.

\subsection{Statistical Analysis}

Patients who were placed on systemic steroid therapy concomitant with the diagnosis of H1N1 influenza pneumonia were compared with those not receiving steroids at the time of diagnosis. A second analysis to determine factors related to mortality in the studied population was performed. In the descriptive analysis percentages were used in categorical variables, mean $\pm \mathrm{SD}$ in continuous variables, and median (range) in the others. Anova method is used to find the association of between the steroid administration and length of hospital stay.

\section{Results}

\section{Age and Sex Distributions}

The mean age of the participants in this study was $27.54 \pm$ 1.67 in which there were 60 males $(25.2 \pm 1.87)$ and 55 females $(30 \pm 1.39)$ in this study. An $8.7 \%$ of the total H1N1 cases and $19 \%$ of the total female (46\%) population was pregnant.

\section{History of Travel}

History of travel to an epidemic region was noticed in $14.8 \%$ of the patients and history of contact noticed in $16.5 \%$ of the patients.

\section{Hospitalisation}

Hospitalization rate was higher in the age group of 19-39 years. $26.1 \%$ of the total cases were in paediatric population and H1N1 cases was noted to be slightly higher in the male sex in the adult population ( male $53.9 \%$ \& female $46.1 \%$ ), as well as in paediatric population ( 22 out of 62 males and 8 out of 53 females).

\section{Symptoms}

Fever (97.4\%) was the most common clinical manifestation followed by cough $(80 \%)$, breathlessness $(40 \%)$, sore throat $(32.2 \%)$ and chills $(20 \%)$ in the patients. Myalgia was present in $19.1 \%$ of the patients, headache $(11.3 \%)$, running nose $(8.7 \%)$, fatigue $(8.7 \%)$, diarrhoea and vomiting $(5.2 \%)$, chest pain (3.5\%), sneezing (3.5\%), redness of the eyes $(1.7 \%)$, and wheezing in $(0.9 \%)$.

\section{Laboratory Investigations}

Laboratoricaly leukopenia (16.5\%), neutrophilia (19.1\%), lymphopenia (13.9\%) and thrombocytopenia of $(9.57 \%)$ were noticed in this study. Other laboratory findings of significance were hyponatremia $(33.9 \%)$, hypokalaemia $(12.2 \%)$, and hypoalbuminemia (32.2\%) and elevated serum creatinine levels of $4.35 \%$. Altered liver parameters like high SGPT in $21.7 \%$, high SGOT in $28.7 \%$, and a total of bilirubin in $8.7 \%$ of the patients were noted at the time of admission. An elevated level of CRP $(>6)$ in $42.6 \%$ and an ESR $(>20)$ in $49.6 \%$ was also noticed in preliminary screening.

ABG was taken from all patients with suspected hypoxemia (Oxygen Saturation ( $\mathrm{SpO} 2$ ) of less than 95\% via pulse oxymetry). Only one patient had a normal ABG, most of the patients showed type I respiratory failure, which was the most difficult problem to tackle in the intensive care unit. An analysis of the x-ray findings of the patients showed a normal study in $70.4 \%$ and bilateral infiltrates in $26.1 \%$. Blood cultures were taken in 30 patients and a significant pathogen was isolated in 5 patients which included Staphylococcus aureus, Pseudomonas and Acinetobacter. Sputum/Endotracheal tube tip cultures were also taken in 24 patients with pneumonia; significant pathogens isolated 
include Klebsiella, Pseudomonas and Acinetobacter. Next most common complication noted was altered liver functions in $8.7 \%$ of the patients; $7 \%$ of the patients had sepsis; $6.1 \%$ had acute renal failure and 5.2\% had MODS.

\section{Treatment}

A total of $91.3 \%$ patients were treated with antibiotics, $80 \%$ patients were treated with antivirals and $27.8 \%$ were treated with steroids. Among the $115 \mathrm{H} 1 \mathrm{~N} 1$ patients $27 \%$ patients required oxygen, $13 \%$ required treatment with non-invasive ventilation (BiPAP), 10.4\% patients needed mechanically ventilation to support the airway and $5.2 \%$ patients treated with inotropic supports.

Corticosteroid therapy was administered to $30(27.8 \%)$ patients. Steroid therapy was initiated within a median of 1 (Interquartile range (IQR), 0-6 day of admission. Steroid therapy was initiated at a median daily dose equivalent to 270 (IQR, 200-400) mg hydrocortisone / Methylprednisolone, and patients were treated for a median duration of 11 (IQR, 5-7) days.

From (TABLE 1) It is inferred that the patient's age and steroid administration have an association since the $\mathrm{p}$ value is less than $0.05(p=0.028)$. But gender does not have any relationship with steroid administration $(\mathrm{p}=0.312)$. The steroid administration also has a strong association with antibiotic $(\mathrm{p}=0.042)$, antiviral $(\mathrm{p}=0.000)$, oxygenation $(\mathrm{p}=$ $0.000)$, mechanical ventilation $(\mathrm{p}=0.000)$, BiPAP $(\mathrm{p}=$ $0.000)$, and mortality $(p=0.001)$ since the $p$ value are less than 0.005 .

Of the $115, \mathrm{H} 1 \mathrm{~N} 1$ confirmed patients $92.6 \%$ patients were admitted as inpatient and 7.4\% patients were treated as outpatient. $93.9 \%$ of the patients improved and were discharged, but $6.1 \%$ of the total $\mathrm{H} 1 \mathrm{~N} 1$ cases and $7.4 \%$ of the total inpatients expired. The reason for mortality noted was severe bronchopneumonia/ARDS with difficulty in ventilation along with secondary infections. In this study age has no association with mortality $(p=0.357)$ whereas gender is related to mortality $(p=0.039)$. The other variables have no relationship with mortality (TABLE 2 ).

Out of 32 patients admitted in ICU $40.6 \%$ required a 3-7 days stay in ICU, 25\% patients required more than 14 days of care in ICU and $28.1 \%$ patients needed only $1-3$ days monitoring in ICU. But a total of 94 patients needed a 3-7 day stay in the ward (including patients shifted from ICU), 24 patients discharged within 1-3 days and 2 patients required more than 14 days stay in the ward and both of them were shifted from ICU after a 7-14 days care.

(From Table 3) Of the 115 patients, 30 patients have been administered steroids. In that, 20 have stayed in the ICU with mean days of $7.45 \pm 5.7$ of which 10 patients stayed in ICU alone and 10 patients stayed in ICU and ward with mean days of $10.3 \pm 6.5$ and $13.6 \pm 7.4$ respectively. 10 patients stayed only in the ward with mean days $4.8 \pm 1.9$. Overall mean hospital stay is $9.8 \pm 6.6$ and it was found that there is a statistical significant relationship between corticosteroid administration and reduced hospital stay $(\mathrm{p}=0.000)$.

\section{Discussion}

The epidemic peaks in from 2009 to 2011 correlated with the seasonal monsoons that prevailed at that time in Kerala. Unlike conventional seasonal flu which commonly affects the extremes of age, H1N1 cases were found to be more common in age groups of 19-35 years. This finding was similar to the data from US, where it was due to pre-existing immunity with the old or similar strains of influenza. The hospitalization rate was also high in the age group of 19-39 years [4], [16]. We identified unusual features of severe disease in the 2009 pandemic compared with most previous well characterized pandemics, including the (probable) H2N2 1890 Russian influenza pandemic, the H2N2 1957 Asian influenza pandemic, and the H3N2 1968 Hong Kong pandemic. In these previous influenza pandemics, an increased predilection for infection among children and young adults has been documented[17].

Along with this data, it has been noticed that women have higher mortality rates more so than males in this study. This is assumed to be due to the fact that these patients were pregnant at the time they were infected with H1N1. These patients were susceptible to complications such as acute respiratory distress syndrome and severe bronchopneumonia.

The level of pulmonary replication of the $2009 \mathrm{H} 1 \mathrm{~N} 1$ virus has been higher than that of seasonal influenza A (H1N1) in experimentally infected animals, but the 2009 pandemic strain generally lacks mutations that are associated with increased pathogenicity in other influenza viruses. Chronic lung disorders such as asthma, COPD, cystic fibrosis are the major risk factors that lead to complications of the 2009 H1N1 virus infection. The main complication is Acute Lung Injury (ALI) or ARDS [4]. Despite advances in antimicrobial therapy, CAP remains a serious illness and is a considerable cause of mortality and morbidity [5]

Corticosteroids are the most effective and widely used anti-inflammatory drugs. The role of corticosteroids as an adjunctive therapy for pneumonia is still under debate. An early study demonstrated the association of glucocorticoids with antibiotics attenuated local inflammatory response and decreased bacterial burden in the experimental model of severe pneumonia. In a mouse pneumonia model, Li et al. found that hydrocortisone decreased inflammatory response significantly. In addition, Salluh et al. reported that relative adrenal insufficiency occurred in most of the patients with severe pneumonia suggesting underlying benefits of corticosteroids treatment in these patients. Taken together, these facts indicated a potential beneficial effect of corticosteroids in pneumonia [6]

Recently, a multicentre randomized controlled trial (RCT) performed by Confalonieri et al. demonstrated that hydrocortisone treatment in severe pneumonia was associated with a significant reduction in mortality. A retrospective study conducted by Garcia-Vidal et al. found that mortality decreased in the patients who received 
systemic steroids along with antibiotic treatment for severe pneumonia. Moreover these results, from a systematic review showed that administration of corticosteroids in patients with pneumonia was associated with a lower mortality [8]. However, these findings were not confirmed in the subsequent larger RCTs [9]-[11]. Another recent retrospective study also showed that adjunctive therapy with corticosteroids did not influence the mortality rate [12].

Furthermore, results from a meta-analysis found that participants receiving corticosteroids displayed no significant differences in mortality compared with a placebo [13]. Consequently, the benefits of corticosteroids treatment in pneumonia are still uncertain. Some clinical trials testing the usefulness of systemic steroids as adjunctive therapy of severe pneumonia have described some benefits by decreasing mortality or length of stay, while others show no benefits in the same end-points or even an increase in related side effects (e.g. hyperglycaemia, super-infections) [14].

A recent meta-analysis does not support the recommendation of corticosteroids as a standard of care for patients with severe pneumonia, due to the lack of strong evidence. Despite providing a clear benefit, the use of corticosteroids in pneumonia is relatively frequent in practice, possibly in relation to patients' comorbidities (e.g. respiratory diseases, cardiac or renal failure) or to clinical presentation (wheezing or rhonchi)[3]

Many critically ill patients during the outbreak also received corticosteroid therapy as a part of management of respiratory failure, ranging from $18 \%$ in the report Australia-New Zealand Intensive Care Society (ANZICS) to $51 \%$ in Canadian experience. These groups of investigators, however, did not comment on the potential effects of corticosteroids in their context. A report on small series of patients with severe influenza pneumonia suggests a beneficial effect of corticosteroids, but registry data from China or Europe suggest possible harm from steroid therapy [15].

The utility of administering corticosteroids in pneumonia is still an unresolved issue in the literature as you can see. Although animal and observational studies on inflammation suggest a potential benefit by decreasing lung bacterial burden and systemic inflammation, evidence in humans is unclear. The current guidelines for CAP management do not suggest the use of corticosteroids, independently of comorbidities and severity [3].

Table 1. Characteristics of Patients With or without Steroid Administration

\begin{tabular}{|c|c|c|c|c|}
\hline CHARACTERISTICS & ALL PATIENTS & NO STEROIDS & STEROIDS & P VALUE \\
\hline AGE & $\begin{array}{c}<20 \text { YEARS }(37) \\
20-40 \text { YEARS }(53) \\
>40 \text { YEARS }(25)\end{array}$ & $\begin{array}{c}<20 \text { YEARS }(33) \\
20-40 \text { YEARS }(34) \\
>40 \text { YEARS }(18)\end{array}$ & $\begin{array}{c}<20 \text { YEARS }(4) \\
20-40 \text { YEARS }(19) \\
>40 \text { YEARS }(7)\end{array}$ & $0.028 *$ \\
\hline GENDER & $\begin{array}{c}60-\text { MALE } \\
55-\text { FEMALE }\end{array}$ & $\begin{array}{c}46-\text { MALE } \\
39-\text { FEMALE } \\
\end{array}$ & $\begin{array}{c}14-\text { MALE } \\
16-\text { FEMALE }\end{array}$ & 0.312 \\
\hline ANTIBIOTIC & 105 & 75 & 30 & $0.042 *$ \\
\hline ANTIVIRAL & 92 & 62 & 30 & 0.000 \\
\hline OXYGENATION & 32 & 11 & 21 & 0.000 \\
\hline MVENT & 12 & 1 & 11 & 0.000 \\
\hline BIPAP & 15 & $3-$ & 12 & 0.000 \\
\hline
\end{tabular}




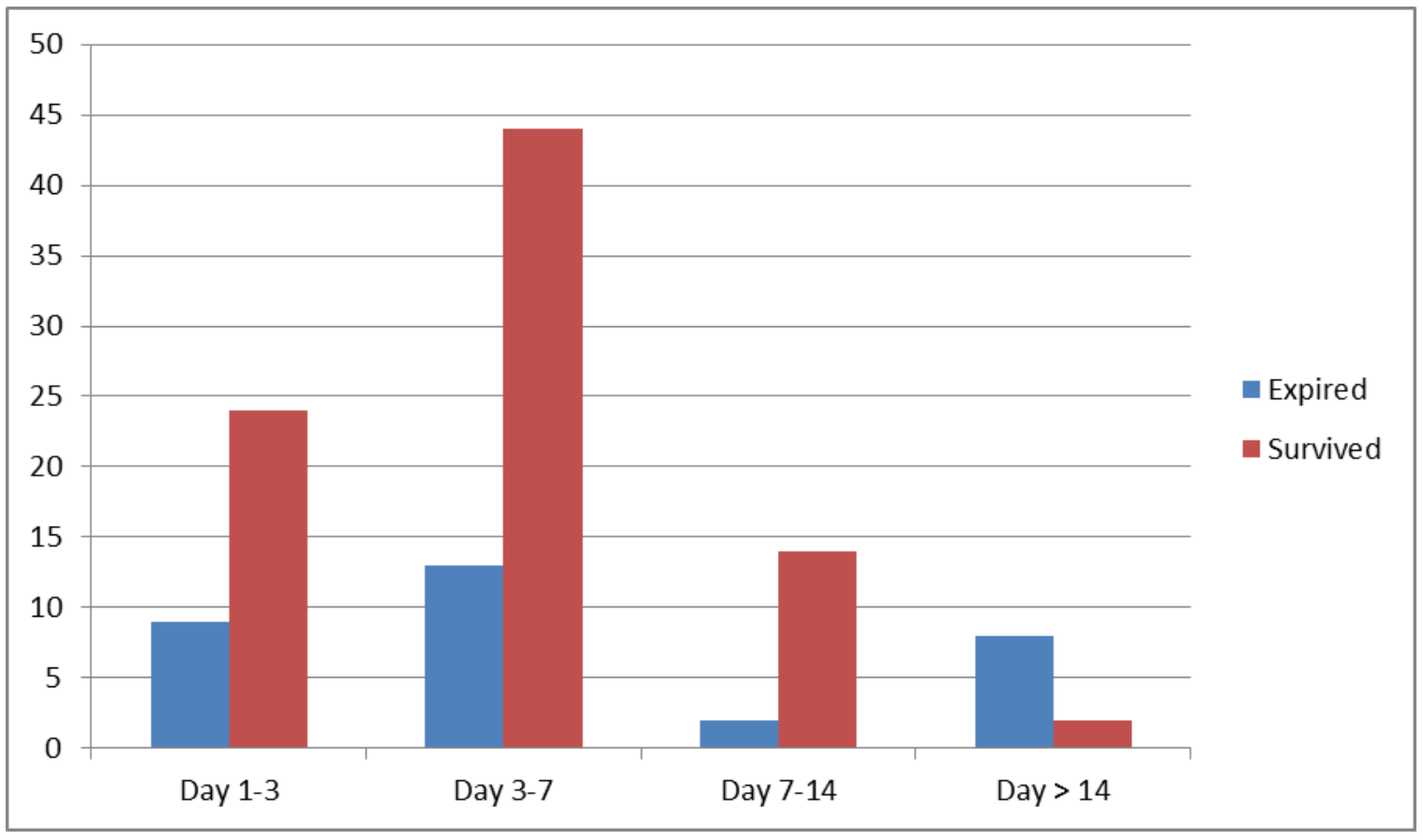

Hospital Stay (number of patients)

\section{Mortality And Morbidity}

Expired - Survived

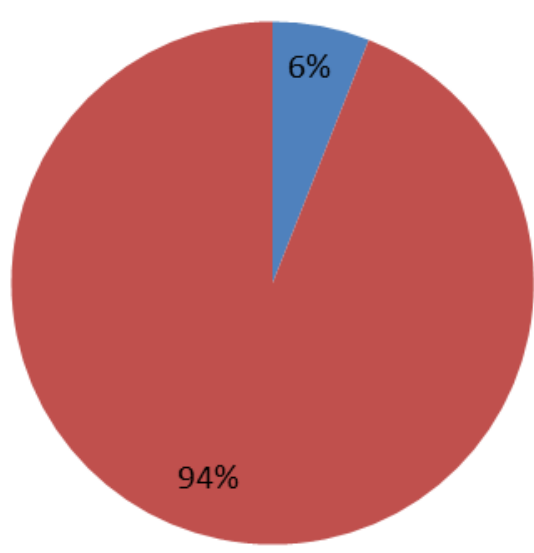

Morbidity and Mortality

\begin{tabular}{|c|c|c|c|}
\hline & H1N1 & IMPROVED (\%) & EXPIRED (\%) \\
\hline TOTAL & 115 & $93.9 \%$ & $7.1 \%$ \\
\hline IP & 95 & $92.6 \%$ & $0 \%$ \\
\hline OP & 20 & $100 \%$ & $30 \%$ \\
\hline PREGNANT & 10 & $70 \%$ & \\
\hline
\end{tabular}


Table 2. Percentage Analysis With P Value Significance

\begin{tabular}{|c|c|c|c|}
\hline CHARACTERISTICS & PERCENTAGE & FREQUENCY & P VALUE \\
\hline AGE i)<20 YEARS & 37 & 32.2 & $0.028^{*}$ \\
ii) $20-40$ YEARS & 53 & 46.1 & 21.7 \\
iii) $>40$ YEARS & 25 & 52.2 & 0.312 \\
\hline GENDER i)MALE & 60 & 97.8 & $0.042^{*}$ \\
\hline ii)FEMALE & 55 & 91.3 & 0.000 \\
\hline ANTIBIOTIC & 105 & 80.0 & 0.000 \\
\hline ANTIVIRAL & 92 & 26.1 & 0.000 \\
\hline STEROIDS & 30 & 27.8 & 0.000 \\
\hline OXYGENATION & 32 & 10.4 & 0.001 \\
\hline M VENT & 12 & 13.0 & 6.1 \\
\hline BIPAP & 15 & & \\
\hline MORTALITY & 7 & & \\
\hline
\end{tabular}

To date, few randomized trials reveal different degrees of CAP severity. The only trial showing reduced mortality was performed in patients admitted in the ICU and the trial was halted prematurely due to $0 \%$ mortality in steroid treatment arm [3]. In contrast, an observational prospective study by Salluh et al. on severe CAP patients described that the main indications for systemic steroids were bronchospasm (52.5\%) and septic shock (36\%). The study found no difference in the mortality in comparison with patients not receiving corticosteroids. Nevertheless, the patients with CAP often receive systemic corticosteroids.

Table 3. Relationship Between Steroid Administration And Length Of Hospital Stay - One Way Anova Method

\begin{tabular}{|c|c|c|c|c|c|}
\hline & $\begin{array}{c}\text { SUM OF } \\
\text { SQUARES }\end{array}$ & df & $\begin{array}{c}\text { MEAN } \\
\text { SQUARES }\end{array}$ & F & $\begin{array}{c}\text { P } \\
\text { VALUE }\end{array}$ \\
\hline $\begin{array}{c}\text { Between } \\
\text { groups }\end{array}$ & 41.691 & 1 & 41.691 & 36.509 & 0.000 \\
\hline $\begin{array}{c}\text { Within } \\
\text { groups }\end{array}$ & 55.956 & 49 & 1.142 & & \\
\hline Total & 97.647 & 50 & & & \\
\hline
\end{tabular}

The incidence of H1N1 cases was more in males among the adult population (males $53.9 \%$ \& females $46.1 \%$ ). A similar trend of male predominance was noticed among the paediatric population as well. Fever was the most common clinical manifestation seen, followed by cough, breathlessness, and sore throat. This was similar to that of US data [18], but cough was the most common symptom in Australia and New Zealand [19].

Most of the patients in this study were treated with antivirals (oseltamivir) along with antibiotics and steroids. Among the 95 admitted patients, $27.8 \%$ needed ICU care and $12.6 \%$ of total admitted patients needed mechanical ventilation. The most common reason for ICU admission was hypoxia and bronchopneumonia/ARDS or viral pneumonitis and later superadded secondary bacterial infection, sepsis, renal failure, and MODS. Hypoxia was the most difficult situation to manage in the ventilated H1N1 patients.

The comorbidities associated with H1N1 cases reported in the US, Australia and New Zealand [19] was bronchial asthma, COPD, and immunosuppression. But in this study it was Type II diabetes mellitus (11.3\%), pregnancy $(8.7 \%)$ and hypertension (6.96\%). Bronchial asthma was noticed only in $3.48 \%$ of the total population in this study. H1N1 infection in pregnancy is associated with a higher mortality and more complications. $18.9 \%$ of the total female population (46.1\%) were pregnant and $30 \%$ of them expired ( 3 out 10 pregnant) due to severe bronchopneumonia/ARDS [23]. The tendency of females to develop severe 2009 influenza A (H1N1) infection in this series is striking. A general female susceptibility has not been observed in other influenza case series of variable severity including the initial reports of 2009 influenza A (H1N1) infections [18]. In most infectious diseases and related conditions such as sepsis and septic shock, males represent a larger proportion of cases and have a higher mortality [20] [21]. The explanation for increased risk of severe disease and death among females in this report is unclear but the role of pregnancy as a risk factor has been noted in previous influenza pandemics [17].

Possible side effects of using corticosteroids, such as increased risk of nosocomial infections, myopathy and hyperglycaemia were not systematically investigated and are not reported in many studies. However, there was no increase in the length of hospital stay as observed. Moreover, Confalonieri et al. prospectively evaluated all patients enrolled in the study for the occurrence of such complications and could not demonstrate an increased risk of these complications in patients using hydrocortisone with placebo [12].

Patients with 2009 influenza A (H1N1) infection-related critical illness experienced symptoms for an average of 4 days prior to hospital presentation, but rapidly worsened and required care in the ICU. The total mortality rate was $6.1 \%$ of the total $\mathrm{H} 1 \mathrm{~N} 1$ confirmed patients, that is, $7.4 \%$ of the total admitted patients. 3 out of 7 expired were pregnant showing a high morbidity and mortality associated with pregnancy [22]. All the mortality was reported in the age group 19-39 years. No mortality was recorded in paediatric and elderly population in this study. Two studies found a significant reduction in mortality for patients with severe pneumonia 
treated with corticosteroids [12]. In the study, Confalonieri et al., a small sized $(n=46)$ multicentre RCT, showed an impressive reduction in hospital mortality $(30 \%$ versus $0 \%$; $=0.009$ ) with a 7 day continuous infusion of hydrocortisone $(240 \mathrm{mg} /$ day). Garcia-Vidal and colleagues evaluated 308 patients in a large retrospective single-centre cohort study and demonstrated in multivariate analysis that the use of corticosteroids was strongly associated with a lower mortality (hospital mortality; odds ratio, $0.287 ; 95 \%$ confidence interval, 0.113 to 0.732 ). These patients were treated with a median dose of $445 \mathrm{mg}$ /day of methylprednisolone for a mean period of 11.4 days. This was done in a small open-label RCT evaluating 31 patients who were receiving a 3 day course of $40 \mathrm{mg}$ of prednisolone as compared with placebo [12].

In this study we found that statistical significant relationship between corticosteroid administration and reduced hospital stay. Similarly, Marik et al. [12] evaluated the effects on clinical course of a single dose of a hydrocortisone administered to patients $(\mathrm{n}=30)$ with severe pneumonia with no increase in length of hospital stay were observed.

\section{Limitations}

There were several limitations to this study. This surveillance system was rapidly established, and the data collection was retrospective from the medical record, where symptoms were not always well documented. This study with H1N1 confirmed patients has not shown much difference from the studies conducted in US, Australia, and even in Chennai. Only a few variations were seen which may be due to regional peculiarities of the population and comorbidities.

\section{Conclusions}

Though we have obtained a statistical significance in the use of corticosteroids and reduced hospital stay on a H1N1 influenza patients we still need a suitable protocol for the use of corticosteroids in CAP. This practice of administering steroids is still relatively frequent with the physician having to make the decision to use these drugs at his own discretion. Only a very well performed, randomized controlled trial focusing on very severe patients will definitely clarify if corticosteroids have some utility in pneumonia despite the possible collateral effects and help draw recommendations and protocol guidelines for clinical practice.

\section{REFERENCES}

[1] Sibila O, Agustí C, Torres A. Corticosteroids in severe pneumonia. Eur Respir J. 2008 Aug;32(2):259-64.
[2] EID Cover - vol15no7_pdf-version.pdf [Internet]. [cited 2015 Feb 1]. Available from: http://wwwnc.cdc.gov/eid/pdfs/voll 5no7_pdf-version.pdf

[3] Systemic corticosteroids for community-acquired pneumonia: reasons for use and lack of benefit on outcome. - PubMed NCBI [Internet]. [cited 2015 Jun 15]. Available from: http://www.ncbi.nlm.nih.gov/pubmed/23134361

[4] Clinical Aspects of Pandemic 2009 Influenza A (H1N1) Virus Infection. N Engl J Med. 2010 May 6;362(18):1708-19.

[5] Efficacy of corticosteroids in the treatment of community-acquired pneumonia requiring hospitalization. PubMed - NCBI [Internet]. [cited 2015 Jun 15]. Available from: http://www.ncbi.nlm.nih.gov/pubmed/17710485

[6] PLOS ONE: Corticosteroids in the Treatment of Community-Acquired Pneumonia in Adults: A Meta-Analysis [Internet]. [cited 2015 Jun 10]. Available from: http://journals.plos.org/plosone/article?id=10.1371/journal.p one.0047926

[7] Severity of community-acquired pneumonia treated with low-dose adjunctive corticosteroid [Internet]. [cited 2015 Jun 10]. Available from:

http://www.ncbi.nlm.nih.gov/pmc/articles/PMC3388627/

[8] Adjunctive therapies for community-acquired pneumonia: a systematic review. - PubMed - NCBI [Internet]. [cited 2015 Jun 15]. Available from:

http://www.ncbi.nlm.nih.gov/pubmed/18641037

[9] Effect of corticosteroids on the clinical course of community-acquired pneumonia: a randomized controlled trial. - PubMed - NCBI [Internet]. [cited 2015 Jun 15]. Available from:

http://www.ncbi.nlm.nih.gov/pubmed/21406101

[10] Efficacy of corticosteroids in community-acquired pneumonia: a randomized double-blinded clinical trial. PubMed - NCBI [Internet]. [cited 2015 Jun 15]. Available from: http://www.ncbi.nlm.nih.gov/pubmed/20133929

[11] Corticosteroids and ICU Course of Community Acquired Pneumonia in Egyptian Settings [Internet]. [cited 2015 Jun 15]. Available from:

http://www.scirp.org/journal/PaperInformation.aspx?PaperI $\mathrm{D}=4454 \#$.VX816i6QqV0

[12] Impact of systemic corticosteroids on the clinical course and outcomes of patients with severe community-acquired pneumonia: a cohort study. - PubMed - NCBI [Internet]. [cited 2015 Jun 10]. Available from: http:/www.ncbi.nlm.nih.gov/pubmed/20889284

[13] Corticosteroids for pneumonia. - PubMed - NCBI [Internet]. [cited 2015 Jun 15]. Available from: http://www.ncbi.nlm.nih.gov/pubmed/21412908

[14] Corticosteroids in the Treatment of Community-Acquired Pneumonia in Adults: A Meta-Analysis [Internet]. [cited 2015 Jun 15]. Available from: http://www.ncbi.nlm.nih.gov/pmc/articles/PMC3480455/

[15] Early Corticosteroids in Severe Influenza A/H1N1 Pneumonia and Acute Respiratory Distress Syndrome (ATS Journals) [Internet]. [cited 2015 Jun 15]. Available from: http://www.atsjournals.org/doi/full/10.1164/rccm.201101-01 35OC\#.VX84xC6QqV0 
[16] Hospitalized Patients with Novel Influenza A (H1N1) Virus Infection --- California, April--May, 2009 [Internet]. [cited 2015 Jun 15]. Available from: http://www.cdc.gov/mmwr/preview/mmwrhtml/mm58e 0518 a1.htm

[17] JAMA Network | JAMA | Critically Ill Patients With 2009 Influenza $\mathrm{A}(\mathrm{H} 1 \mathrm{~N} 1)$ Infection in Canada [Internet]. [cited 2015 Jun 15]. Available from: http://jama.jamanetwork.com/article.aspx?articleid=184799

[18] Estimating the burden of 2009 pandemic influenza A (H1N1) in the United States (April 2009-April 2010). - PubMed NCBI [Internet]. [cited 2015 Jun 15]. Available from: http://www.ncbi.nlm.nih.gov/pubmed/21342903

[19] Clinical and epidemiological profile of patients with severe H1N1/09 pandemic influenza in Australia and New Zealand: an observational cohort study. - PubMed - NCBI [Internet]. [cited 2015 Jun 15]. Available from: http://www.ncbi.nlm.nih.gov/pubmed/22021761

[20] Clinical features of the initial cases of 2009 pandemic influenza A (H1N1) virus infection in China. - PubMed NCBI [Internet]. [cited 2015 Jun 15]. Available from: http://www.ncbi.nlm.nih.gov/pubmed/20007555

[21] Use of Influenza A (H1N1) 2009 Monovalent Vaccine [Internet]. [cited 2015 Jun 15]. Available from: http://www.cdc.gov/mmwr/preview/mmwrhtml/rr58e0821a1 .htm

[22] Case series study of the clinical profile of H1N1 swine flu influenza. - PubMed - NCBI [Internet]. [cited 2015 Jun 15]. Available from:

http://www.ncbi.nlm.nih.gov/pubmed/21751659

[23] 2B-2009-Pandemic-Influenza-A-H1N1.pdf [Internet]. [cited 2015 Jul 28]. Available from: http://healthsciences.ac.in/oct-dec-12/downloads/2B-2009-P andemic-Influenza-A-H1N1.pdf

[24] H1N1 2009 influenza virus infection during pregnancy in the USA. - PubMed - NCBI [Internet]. [cited 2015[Jul 28]. Available from: http://www.ncbi.nlm.nih.gov/pubmed/19643469
[25] CDC protocol of real time RT-PCR for influenza A (H1N1).WHO.

http://www.who.int/csr/resources/publications/swineflu/CD CReal timeRTPCR-wineH1 Assay 2009

[26] Centre for Disease Control and Prevention. Real time RT-PCR (rRTPCR) Protocol for Detection and Characterization of Swine Influenza (version 2009) Available from: http://www.who.int/csr/resources/publicat.

[27] Gandhoke I, Rawat D S, Rai A, Khare S, Ichhpujani R L.Pandemic Influenza A (H1N1) 2009 in India: Duration of virus shedding in patients under antiviral treatment. Indian $\mathbf{J}$ Med Microbiol 2011; 29:37-41.

[28] WHO. Strategic advisory group of experts on immunization-report of the extraordinary meeting on the influenza A (H1N1) 2009 pandemic, 7 July 2009. WklyEpidemiol Rec. 2009; 84:301-304.

[29] Presanis AM, DeAngelis D; New York City Swine Flu Investigation Team, Hagy A, Reed C, Riley S,CooperBS, Finelli L, Biedrzycki P, Lipsitch M. The severity of pandemic H1N1 influenza in the United States, from April to July 2009: A bayesian analysis. PLoS Med. 2009;6:e1000207.

[30] Hollmann M, Garin O, Galante M, Ferrer M, Dominguez A, Alonso J. Impact of influenza on health-related quality of life among confirmed (H1N1)2009 patients. PLoS One. 2013; 8:e60477.

[31] Girard MP, Tam JS, Assossou OM, Kieny MP. The 2009 A (H1N1) influenza virus pandemic: A review. Vaccine.2010; 28:4895-4902.

[32] Bishop JF, Murnane MP, Owen R. Australia's winter with the 2009 pandemic influenza A (H1N1) virus. N Engl J Med. $2009 ; 361: 2591-2594$.

[33] Libster R, Bugna J, Coviello S,et al. Pediatric hospitalizations associated with 2009 pandemic influenza A (H1N1) in Argentina. N Engl J Med. 2010; 362:45-55.

[34] Influenza A H1N1 Pandemic Strain Evolution - Divergence and the Potential for Antigenic Drift Variants Eili Y. Klein, Adrian W. R. Serohijos, Jeong-Mo Choi, Eugene I. Shakhnovich, Andrew Pekosz 